\title{
Radar Imaging of The Target Inside A Dielectric Box
}

\author{
Zhijie Xie, Hui Yue, Zichang Liang
}

Science and Technology on Electromagnetic Scattering Laboratory, Shanghai, 200438, China

Keywords: shooting and bouncing rays; radar cross section; radar imaging; dielectric box

\begin{abstract}
A new algorithm based on shooting and bouncing rays is introduced in this paper. The proposed method is able to achieve the radar cross section (RCS) and radar image of the target inside the dielectric box. A model of dihedral corner reflectors inside a dielectric box is built in this paper, and the RCS and the radar image is calculated by the proposed method.
\end{abstract}

\section{Introduction}

The methods for radar cross section (RCS) calculation of complex targets made of electric conductor are becoming more and more consummate during these years. However, for targets structured by both electric conductor and dielectric materials, the RCS prediction methods are awaiting progress.

In this paper, the transmission calculation is introduced into shooting and bouncing rays (SBR), and then a new algorithm to calculate the RCS and radar image of targets inside dielectric is developed. The SBR method is a high frequency asymptotic method. It was first introduced by $\mathrm{H}$. Ling to calculate the radar cross section of cavities [1], and later extended to arbitrarily shaped targets [2]. The procedure for SBR method can be broken down in three major steps [3]: the raytracing step, the field tracking step and the physical optics step.

In this paper, the dielectric box is designated as transparency, and the electric conductor is holophote in the ray-tracing step. In the field tracking step, the principle of transmission is introduced to calculate the filed in the back of dielectric, and the principles of geometrical optics (GO) is used when the ray meets the electric conductor. The physical optics (PO) is applied to calculate the scattering filed from the exit points in the physical optics step.

\section{The RCS calculation method for targets inside the dielectric box}

This section discusses the method for RCS calculation of targets inside the dielectric box. This method is based on shooting and bouncing rays.

The ray tracing.First in the ray-tracing step, import the geometry information of the target into the software, and then designate the dielectric box as transparency, and the electric conductor as holophote.

Before ray tracing, create a virtual aperture, which is perpendicular to the incident direction [4]. This aperture is divided into dense grids. GO rays are shot into the target area in order to model the incident plane wave. Each ray is represented by a line starting at the aperture and along the incident direction. Once the incident rays have been defined, the impact points of each ray on the object surface can be determined. The ray transmits through the dielectric box, reflects by the electric conductor. The reflection rays can be calculated by the Snell's law. Consequently, by using the reflected ray as the incident ray, the above procedure can be repeated until the ray exits the target area.

Fig. 1 is a demonstrative illustration of ray tracing. The left of the figure is the virtual aperture. It is divided into dense grids. In Fig.1, a ray is emitted from the aperture, and impact the dielectric box at the point $\mathrm{P} 1$. The transmitted ray starts at the point $\mathrm{P} 2$, and reflects at the point $\mathrm{P} 3$ on the electric conductor. The reflected ray by Snell's law hits the electric conductor at the point P4. Then the new reflected ray from point P4 transmits through the dielectric box, and returns to the aperture. This is the procedure of ray tracing for a ray with trace level 2. 


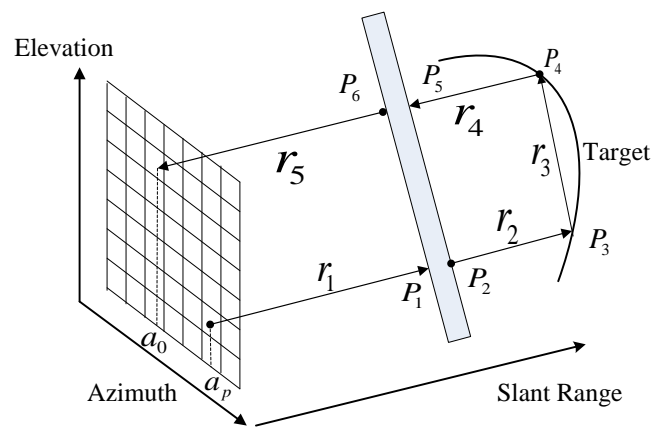

Fig.1: Schematic of ray tracing

The EM field tracking.The information of impact points is obtained in the ray-tracing step. Fig. 2 shows the impact points when the ray incidents into the dihedral corner reflector inside a dielectric box.

Seen from Fig.2, a representative ray has six impact points with the object structured by the dihedral corner reflector and the dielectric box. The purpose of the EM field tracking is to obtain the electric field at the point ${ }^{P_{6}}$.

The incident field at the point $P_{1}$ is determined by the polarization mode. The incident field is [5]

$$
\mathbf{E}_{P_{1}}^{i}=\left[\begin{array}{lll}
0 & E_{\theta} & E_{\varphi}
\end{array}\right] \cdot \mathbf{R T S} \cdot\left[\begin{array}{c}
\hat{\mathbf{x}} \\
\hat{\mathbf{y}} \\
\hat{\mathbf{z}}
\end{array}\right]
$$

where RTS is the transform matrix from the Cartesian coordinates to the spherical coordinates, $E_{\theta}$ and $E_{\varphi}$ are the incident electric fields in the spherical coordinates. For the vertical polarization mode, the electric field is $E_{\theta}=1, E_{\varphi}=0$. For the horizontal polarization mode, the electric field is $E_{\theta}=0, E_{\varphi}=1$.

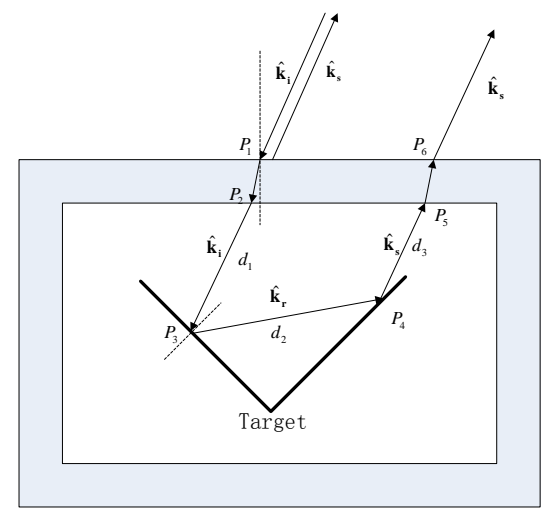

Fig.2: Schematic of impact points

The propagation equation for plane wave in free space is

$$
\mathbf{E}_{\mathbf{B}}=\mathbf{E}_{\mathbf{A}} \cdot e^{-j k l}
$$

where $\mathbf{E}_{\mathbf{A}}$ is the electric field at point $\mathrm{A}, \mathbf{E}_{\mathbf{B}}$ is the electric field at point $\mathrm{B}, k$ is the wave number, $l$ is the distance that electric field travels from point A to point B. According to (2), the electric flied changes can be calculated when the plane wave is propagated from one point to another such as $P_{2} \rightarrow P_{3}, P_{3} \rightarrow P_{4}, P_{4} \rightarrow P_{5}$.

When the electric wave transmits through the dielectric material at points $P_{1}$ and $P_{5}$, the transmitted filed can be writing as

$$
\mathbf{E}^{\mathbf{t}}=\left(\mathbf{E}^{\mathbf{i}} \cdot \hat{\mathbf{e}}_{\perp}\right) \dot{T}_{i}^{\perp} \hat{\mathbf{e}}_{\perp}+\left(\mathbf{E}^{\mathbf{i}} \cdot \hat{\mathbf{e}}_{\mathbf{p}}\right) \dot{T}_{i}^{p} \hat{\mathbf{e}}_{\mathbf{p}}
$$


where $\mathbf{E}^{\mathbf{i}}$ is the incident electric field, $\hat{\mathbf{e}}_{\perp}$ and $\hat{\mathbf{e}}_{\mathbf{p}}$ are the unit vectors of the vertical and parallel modes, $\dot{T}_{i}^{\perp}$ and $\dot{T}_{i}^{p}$ are the transmission coefficients for vertical and parallel modes. Transmission coefficient can be write as

$$
\dot{T}=\frac{2}{2 \cosh \left(j \gamma_{1} d\right)+\left(Z_{01}+\frac{1}{Z_{01}}\right) \sinh \left(j \gamma_{1} d\right)}=T_{0} \exp \left(-j \varphi_{t}\right)
$$

where $\gamma_{1}=2 \pi \sqrt{\dot{\varepsilon}_{r}-\sin ^{2} \theta_{i}} / \lambda$ is the propagation factor in the dielectric; $d$ is the thickness of the dielectric box; $\dot{\varepsilon}_{r}$ is the permittivity of the dielectric; $\theta_{i}$ is the incident angle; $Z_{01}$ is the uniformed impedance, it is

$$
Z_{01}=\left\{\begin{array}{l}
Z_{p}=\sqrt{\dot{\varepsilon}_{r}-\sin ^{2} \theta_{i}} /\left(\dot{\varepsilon}_{r} \cos \theta_{i}\right) \\
Z_{\perp}=\cos \theta_{i} / \sqrt{\dot{\varepsilon}_{r}-\sin ^{2} \theta_{i}}
\end{array}\right.
$$

where $Z_{p}$ and $Z_{\perp}$ stands for the uniformed impedances of parallel mode and vertical mode.

In high frequency situation, the reflection of electromagnetic waves on the target surface obeys the algorithm of geometrical optics. According to the Snell's reflection law and the boundary conditions, the reflected field at points $P_{3}$ and $P_{4}$ can be expressed as

$$
\mathbf{E}^{\mathbf{r}}=\mathbf{2}\left(\hat{\mathbf{n}} \cdot \mathbf{E}^{\mathrm{i}}\right) \cdot \hat{\mathbf{n}}-\mathbf{E}^{\mathbf{i}}
$$

where $\hat{\mathbf{n}}$ is the normal vector of the reflection point, $\mathbf{E}^{\mathbf{i}}$ is the electric field of the incident wave, $\mathbf{E}^{\mathbf{r}}$ is the electric field of the reflected wave.

Applying to the above method, the electric field at exit point of each ray can be obtained.

The scattering field calculation.Seen from Fig.2, the total scattering field can be break into two main parts, one is the transmission field from the dihedral corner reflector; the other is the reflection field from the dielectric box.

This paper adopts the method of PO [5] in the final scattered field computation. PO is a typical high-frequency asymptotic method. This method uses the electromagnetic current on the surface instead of the scatter, and integrates the induction field of the current to obtain the scattered field.

For the transmission field calculation at the point ${ }^{P_{6}}$, the induced current can be expressed as

$$
\mathbf{J}\left(\mathbf{r}^{\prime}\right)=2 \hat{\mathbf{n}} \times \mathbf{H}_{\mathrm{i}}\left(\mathbf{r}^{\prime}\right)
$$

where $\hat{\mathbf{n}}$ is the normal vector, $\mathbf{H}_{\mathbf{i}}\left(\mathbf{r}^{\prime}\right)$ is the incident magnetic field at the exit point, $\mathbf{r}^{\prime}$ is the position vector of the exit point.

According to Kirchhoff's law, the transmission field contribution can be obtained by accumulating the field contribution of each transmitted ray [6]:

$$
\mathbf{E}_{\mathbf{s t}}(\mathbf{r}) \cong \frac{j k}{4 \pi} \cdot \frac{e^{-j k R}}{R} \cdot \int_{S_{c}} \hat{\mathbf{s}} \times\left(Z_{0} \hat{\mathbf{s}} \times \mathbf{J}\left(\mathbf{r}^{\prime}\right)\right) \exp \left(j k \mathbf{r}^{\prime} \cdot \hat{\mathbf{s}}\right) d S_{c}^{\prime}
$$

where $\hat{\mathbf{s}}$ is the unit vector of the scattering direction, $Z_{0}$ is the wave impedance in free space, $R$ is the distance from the target to the antenna, and $\mathbf{r}^{\prime}$ is the position vector of the observation point. In this paper, (8) can be solved by the Gordon's method [6, 7].

For the reflection field calculation at the point ${ }^{P_{1}}$, the reflection coefficient is[8]

$$
\dot{\Gamma}=\frac{\left(Z_{01}-\frac{1}{Z_{01}}\right) \sinh \left(j \gamma_{1} d\right)}{2 \cosh \left(j \gamma_{1} d\right)+\left(Z_{01}+\frac{1}{Z_{01}}\right) \sinh \left(j \gamma_{1} d\right)}
$$

where the parameters in (9) are the same with those in (4).

The induced magnetic current and electric current of the reflected field can be expressed as

$$
\mathbf{J}_{\mathbf{H}}=\left(1+\dot{\Gamma}_{\perp}\right)\left(\hat{\mathbf{e}}_{\perp} \cdot \mathbf{E}^{\mathbf{i}}\right) \hat{\mathbf{n}} \times \hat{\mathbf{e}}_{\perp}+\left(1-\dot{\Gamma}_{p}\right)\left(\hat{\mathbf{e}}_{\mathbf{p}} \cdot \mathbf{E}^{\mathbf{i}}\right)(\hat{\mathbf{n}} \cdot \hat{\mathbf{i}}) \hat{\mathbf{e}}_{\perp}
$$




$$
\mathbf{J}_{\mathbf{E}}=\left[-\left(1-\dot{\Gamma}_{\perp}\right)\left(\hat{\mathbf{e}}_{\perp} \cdot \mathbf{E}^{\mathbf{i}}\right)(\hat{\mathbf{i}} \cdot \hat{\mathbf{n}}) \hat{\mathbf{e}}_{\perp}+\left(1+\dot{\Gamma}_{p}\right)\left(\hat{\mathbf{e}}_{\mathbf{p}} \cdot \mathbf{E}^{\mathbf{i}}\right) \hat{\mathbf{n}} \times \hat{\mathbf{e}}_{\perp}\right] / Z
$$

where $\dot{\Gamma}_{p}$ and $\dot{\Gamma}_{\perp}$ are the reflection coefficients for the parallel and vertical mode; $\hat{\mathbf{i}}$ is the unit vector of the incident direction.

The reflection field contribution is

$$
\mathbf{E}_{\mathbf{s r}}=\frac{j k e^{-j k R}}{4 \pi R} \int_{s} \hat{\mathbf{s}} \times\left[\mathbf{J}_{\mathbf{H}}-Z_{0} \hat{\mathbf{s}} \times \mathbf{J}_{\mathbf{E}}\right] e^{j k \mathbf{r}^{\prime} \cdot \hat{s}} d s
$$

The total scattering field can be write as

$$
\mathbf{E}_{\mathrm{s}}=\mathbf{E}_{\mathrm{st}}+\mathbf{E}_{\mathrm{sr}}
$$

$\mathbf{E}^{\mathbf{s}}$ is the total scattering field in the Cartesian coordinates. The scattering field received by the radar receiver is determined by the polarization mode, and the polarization is decided in the spherical coordinates. The scattering field in the spherical coordinates is

$$
\mathbf{E}^{\mathbf{s}}=\mathbf{E}^{\mathrm{s}} \cdot \mathbf{S T R} \cdot\left[\begin{array}{l}
\hat{\mathbf{r}} \\
\hat{\boldsymbol{\theta}} \\
\hat{\boldsymbol{\varphi}}
\end{array}\right]=\left[\begin{array}{lll}
0 & E_{\theta}^{s} & E_{\varphi}^{s}
\end{array}\right] \cdot\left[\begin{array}{l}
\hat{\mathbf{r}} \\
\hat{\boldsymbol{\theta}} \\
\hat{\boldsymbol{\varphi}}
\end{array}\right]
$$

where STR is the transform matrix from the spherical coordinates to the Cartesian coordinates, $E_{\theta}^{s}$ and $E_{\varphi}^{s}$ are the scattering fields in the spherical coordinates. The scattering field by the radar receiver is

$$
\mathbf{E}_{\mathbf{o}}= \begin{cases}E_{\theta}^{s} \cdot \hat{\boldsymbol{\theta}} & V \text { polarization } \\ E_{\varphi}^{s} \cdot \hat{\boldsymbol{\varphi}} & H \text { polarization }\end{cases}
$$

When the scattering filed by the radar receiver is obtained by (15), the RCS can be expressed as

$$
\sigma=4 \pi \lim _{r \rightarrow \infty} r^{2} \frac{\left|\mathbf{E}_{o}\right|^{2}}{\left|\mathbf{E}_{i}\right|^{2}}
$$

where $\mathbf{E}_{\mathbf{i}}$ is the incident field at the target.

\section{Computational examples}

The high resolution range profile of the target inside a dielectric box.The first example is the RCS and high-resolution range profile (HRRP) calculation of the dihedral corner reflector inside a dielectric box. The model is shown in Fig.3. The right-angle side length of the dihedral corner reflector is $1 \mathrm{~m}$. The size of the dielectric box is $3 \mathrm{~m}$, and the thickness of the dielectric box is $0.05 \mathrm{~m}$.

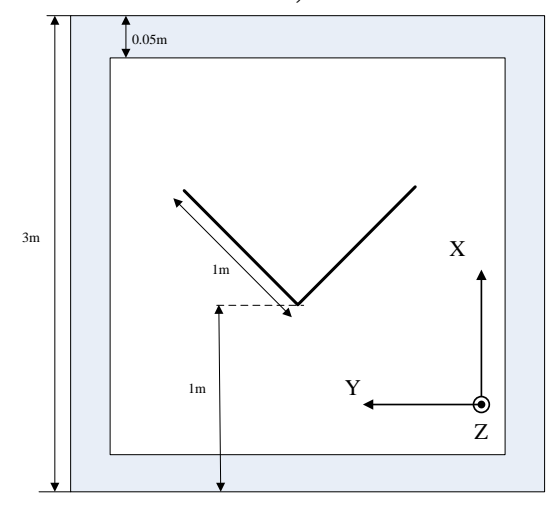

Fig.3: Dihedral corner reflector inside a dielectric box

In the example, the elevation angle is $0^{\circ}$ (the angle between the radar line of sight and XOY plane). The azimuth angle is $0^{\circ}$ (the angle between the projection of radar line of sight on XOY plane and the X-axis). The frequency sweeps from $1 \mathrm{GHz}$ to $10 \mathrm{GHz}$ with a step of $20 \mathrm{MHz}$. $\mathrm{HH}$ polarization is used in the calculation. The permittivity of the dielectric box is $\dot{\varepsilon}_{r}=4.0-0.5 i$. The 
HRRP can be achieved by inverse Fourier transforming the computed scattered fields over frequency domain. The result is shown in Fig.4.

In Fig.4(a), the RCS increases along with the frequency. The HRRP shown in Fig.4(b) has four scattering centres. A stands for the scattering centre of the dielectric board, B is the virtual image of the dielectric board, $\mathrm{C}$ stands for the scattering centre of the dihedral corner reflector, and $\mathrm{D}$ is the virtual image of the dihedral corner reflector.
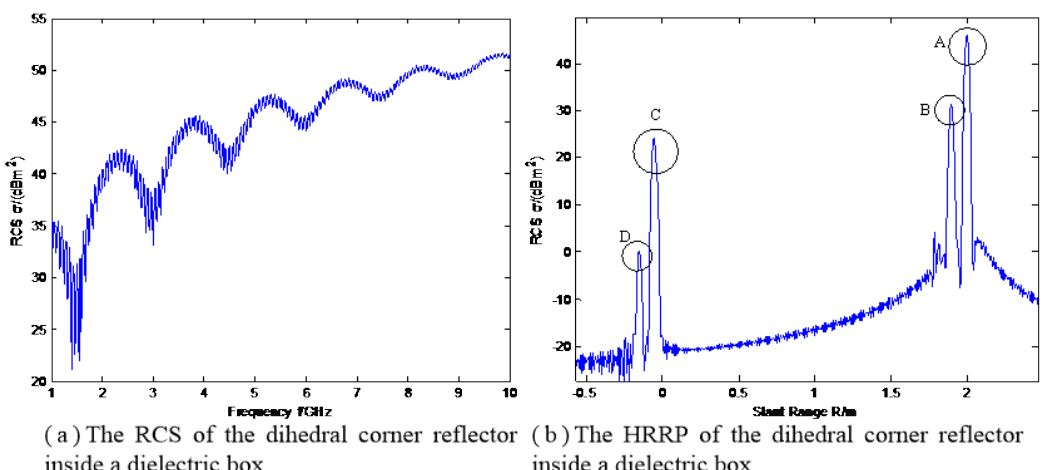

Fig.4: The RCS and HRRP of the dihedral corner reflector inside a dielectric box

The radar imaging of the target inside the dielectric box.In the following example, the radar image of the model shown in Fig. 3 is studied. The scattered fields of the model are calculated by the proposed method described in section II first. The radar image is generated by inverse Fourier transforming the computed scattered fields over frequency and aspect domain [9].

In this example, the model is calculated with a center frequency of $5 \mathrm{GHz}$. The bandwidth is $6 \mathrm{GHz}$, and the frequency increment is $20 \mathrm{MHz}$. The elevation angle is $0^{\circ}$. The azimuth angle sweeps from $-8.6^{\circ}$ to $8.6^{\circ}$ with a step of $0.172^{\circ}$. $\mathrm{HH}$ polarization is used in the calculation. The permittivity of the dielectric box is $\dot{\varepsilon}_{r}=4.0-0.5 i$. In Fig.5, the result is compared with the radar image of the dihedral corner reflector.

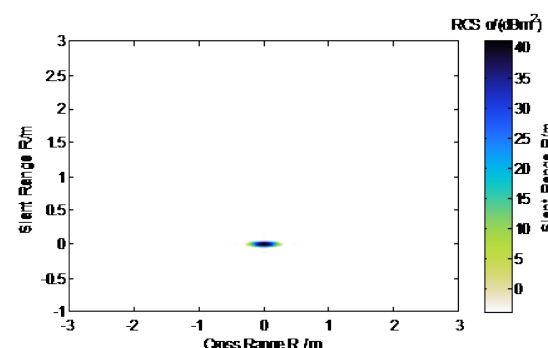

(a) The radar image of the dihedral corner reflector

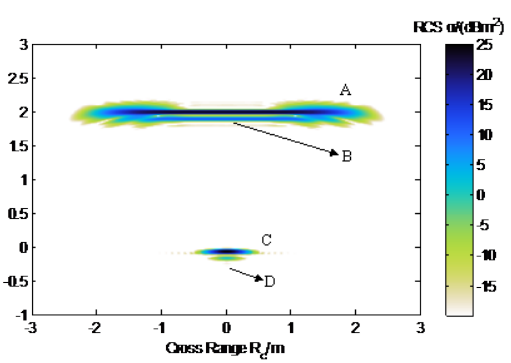

(b) The radar image of the dihedral corner reflector inside a dielectric box

Fig.5: Radar image

The Fig.5(a) is the radar image of the dihedral corner reflector using the same parameters. The Fig.5(b) is the result of dihedral corner reflector inside a dielectric box by the proposed method. In the Fig.5(b), A stands for the scattering centre of the dielectric board, B is the virtual image of the dielectric board, $\mathrm{C}$ stands for the scattering centre of the dihedral corner reflector, and $\mathrm{D}$ is the virtual image of the dihedral corner reflector.

\section{Summary}

In this paper, a new procedure in RCS calculation and radar image formation for targets inside the dielectric box based on SBR is introduced. The proposed method is used to calculate the RCS and the radar image of the dihedral corner reflectors inside the dielectric box. 


\section{Acknowledgements}

The research work was supported by National Natural Science Foundation of China under Grant No. 61302036.

\section{References}

[1] H. Ling, R. C. Chou, and S. W. Lee, Shooting and Bouncing Rays: Calculating the RCS of an arbitrarly shaped cavity, IEEE Transactions on Antennas and Propagation, vol. 37, No. 2, pp.194-205, Feb. 1989.

[2] J. Baldauf, S. W. Lee, L. Lin, S. K. Jeng, S. M. Scarborough, C. L. Yu. High frequency scattering from trihedral comer reflectors and other benchmark targets: SBR VS experiments, IEEE Transactions on Antennas and Propagation, vol. 39, No. 9, pp.1345-1351, 1991.

[3] Z. J. Xie. and X. J. Xu, Radar cross section calculations and radar image formations using shooting and bouncing rays, 2013 International Coferance on Image and Signal Procesing, Dec. 2013.

[4] S. Auer, S. Hinz, and R. Bamler, Ray-tracing simulation techniques for understanding highresolution SAR images, IEEE Transactions on Geoscience Remote Sensing, vol. 48, No. 3, pp. 1445-1456, Mar 2010.

[5] M. Sui, PO Source Code, Beijing: Remote Sencing Signatures Lab (F403) in Beihang University, Oct. 2010.

[6] C. Wang, High Frequency electromagnetic scattering modeling and its applications. Beijing: Communication University of China, 2009.

[7] W. B. Gordon, Far-field approximations to the Kirchhoff-Helmholtz representations of scattered fields, IEEE Transactions on Antennas and Propagation, vol. 23, No. 5, pp. 590-592, Jul 1975.

[8] Z. Zhang, L. Jin, The Technique of Radar Antennas. Beijing: Publishing House of Electronics Industry, 2010.

[9] X. Xu, P. Huang, Radar System and Signal Processing. Beijing: Publishing House of Electronics Industry, 2010. 